\title{
PROFILE OF NEONATAL ADMISSION AT CHITWAN MEDICAL COLLEGE
}

\author{
SP Shrestha ${ }^{1 *}$, AK Shah $^{2}$, R Prajapati ${ }^{3}$, YR Sharma $^{3}$ \\ ${ }^{1}$ Department of Pediatrics, Chitwan Medical College, Bharatpur-10, Chitwan, Nepal. \\ ${ }^{2}$ Department of Microbiology, Chitwan Medical College, Bharatpur-10, Chitwan, Nepal. \\ ${ }^{3}$ Department of Pediatrics, Chitwan Medical College, Bharatpur-10, Chitwan, Nepal. \\ *Correspondence to : Dr Shiva Shrestha, HoD, Department of pediatrics, Chitwan Medical College, Bharatpur-10, Chitwan, Nepal. \\ Email: dr shrestha shivanita@yahoo.com
}

\begin{abstract}
In Nepal, three most common causes of newborn (NB) admission in the neonatal intensive care unit (NICU) are birth asphyxia, neonatal sepsis and prematurity and they are the leading causes of death too. A study previously done in Nepal shows asphyxia as a leading cause of hospital admission accounting $22 \%$ followed by prematurity $20 \%$ and neonatal sepsis $17 \%$ with mortality due to these three causes being 7\%, 3\% and 5\% respectively. Reasons of NICU admission in Chitwan Medical College (CMC) are clinical sepsis in $50 \%$ of cases followed by birth asphyxia $17.6 \%$, and prematurity in $6.7 \%$ cases. Respiratory distress syndromes (RDS), neonatal jaundice, congenital hydrocephalus, meningitis are other reasons of NICU admission. Most of the babies were delivered by normal vaginal delivery at health facility $53 \%$, and $47 \%$ of NB was delivered through lower section Caesarean section (LSCS). Only 6\% of NB was having birth weight of less than $1.5 \mathrm{~kg}$ and majority of them were above that. There were $65 \%$ NB who was born at term and remaining 35\% were preterm. Majority of NB were admitted within 72 hours of birth with $79 \%$ of the total admission. Hypoglycemia was observed in 83 out of 202 newborns accounting $41 \%$ neonatal hypoglycemia at birth.
\end{abstract}

Key Words: Asphyxia, Hypoglycemia, Newborn, NICU, Prematurity, RDS.

\section{INTRODUCTION}

Established 6 years ago by a group of young, energetic and enthusiastic Nepalese doctors, Chitwan Medical College (CMC) aims of providing best health services to the Nepalese people, producing competent health manpower that is required for the nation and conducting research activities in the related disciplines. CMC provides neonatal services right from the establishment of this institute to cater highly specialized neonatal services to the sick and needy newborns. Neonatal sepsis has been one of the frequent causes of neonatal Intensive care unit (NICU) admission since then. This study aims to study its profile of hospital admission, disease patterns and its prevalence.

Neonatal sepsis has been one of the major reasons of admission to the neonatal units worldwide and neonatal sepsis is one of the commonest causes of neonatal admission in more than fifty percent of cases at NICU unit of CMCTH. Neonatal sepsis (NS) is one of the major causes of morbidity and mortality among the newborns in the developing world ${ }^{1}$ too. Neonatal mortality rates in Nepal as per NDHS 2011 data is 33 per 1000 live births. $^{2}$

Despite the development of newer and more potent antimicrobials, sepsis is still a major threat of morbidity and mortality among the newborns. There are many reasons for sepsis in new born period. Some of the factors responsible are immaturity of immune system which includes decreased phagocytic activity of white blood cells, decreased production of cytokines and weak cellular and humoral immunity. Infection can occur either in intrauterine period, during delivery or after the baby is born. It can also occur from the community or from the hospital itself. However, most of the causes of neonatal morbidity and mortality are preventable. ${ }^{3}$

In Nepal, three most common causes of neonatal admission in the NICU unit are birth asphyxia, neonatal sepsis and prematurity and are also the leading causes of death. A study previously done in Nepal shows asphyxia as a leading cause of hospital admission (22\%) followed by prematurity (20\%) and neonatal sepsis $(17 \%)$ with mortality due to these three causes being $7 \%$, $3 \%$ and $5 \%$ respectively. ${ }^{4}$ So, among them neonatal sepsis is one of the major causes of morbidity and mortality. The risk of new born dying is 24 per 1000 live births in the first week of life, 3 per 1000 per week during the rest of the first month and 0.12 per 1000 per week after the first year of life..$^{5}$

It is not easy to diagnose sepsis in neonatal period and high index of suspicion is required for its early diagnosis. It is estimated that $20 \%$ of all neonate develop sepsis. ${ }^{6}$ The reported incidence of NS varies from 7.1 to 38 per 1000 live births in Asia. ${ }^{7}$ There are multiple causes that contribute to neonatal sepsis. Those factors responsible for NS are preterm delivery, IUGR, birth asphyxia, prolonged rupture of membrane lasting more than 18 hours, 
maternal fever, choreo-amnionitis, muconium stained liquor, baby needing active resuscitation and unhygienic delivery practices like unsafe delivery by traditional birth attendants.

Neonatal sepsis (NS) may acquire during intrauterine period, during delivery time or after delivery at nursery itself. NS is broadly classified according to its onset; Early onset neonatal sepsis (EONS) occur within $(<72 \mathrm{hrs})$ and late onset neonatal sepsis (LONS) after ( $>72 \mathrm{hrs}$ to 28 days). This classification is of clinical importance since pneumonia is more common in EONS and septicemia and meningitis are more common in LONS. So as, organisms causing sepsis also do differ.

Common bacteria that are responsible for EONS are E.coli, Klebsiella, Proteus, Pseudomonas, Staphylococcus and Listeria monocytogens. Group B streptococcus is not the cause of sepsis in developing countries like Nepal. LONS is most commonly caused by Coagulase-negative staphylococci (CONS), Staphylococcus aureus, Escherichia coli, Klebsiella and Pseudomonas and is usually acquired in the NICU or the community. ${ }^{8}$

Neonates do not exhibit the features of sepsis of systemic involvement as we expect. So, already mentioned that high clinical suspicion and judgment is mandatorily required. Clinically, newborns are suspected of having sepsis if they present with lethargy, poor tone, poor cry, refusal to feed or poor feeding, temperature instability (hypo or hyperthermia), vacant stare, respiratory distress, shock, circulatory collapse, abdominal distension and frequent vomiting after each feed.

\section{MATERIALS AND METHODS}

This is a hospital based retrospective study conducted in the neonatal unit (NICU) of Chitwan Medical College and Teaching Hospital (CMCTH) over a period of one year. CMCTH is delivering tertiary level health services to its citizen besides providing Clinical, Preventive and Nursing education at master's level.

Data files of NICU admitted infants were retrieved from the record section of the $\mathrm{CMCTH}$ and microbiology department and analyzed for the study. There were total of 270 neonates admitted in the neonatal ward during the period of one year 1st of Baisakh 2069(April 13, 2012) to end of Chaitra 2069(13 of April 2013) and data was analyzed in 267 newborns only since all necessary data were not available in rest of the 3 cases. Majority of admitted cases were of clinical sepsis accounting $50.18 \%$ of the total neonatal admission. The neonates were suspected of having sepsis if they presented with one of the following signs or symptoms like; lethargy, refusal to feed or poor feeding, temperature instability (hypo or hyperthermia), vacant stare, poor tone, poor cry, respiratory distress, jaundice, shock, circulatory collapse, sclerema neonatorum, abdominal distension and frequent vomiting after each feed and others.

Septic screening was done in all clinically suspected sepsis. Complete blood counts (that includes TLC, DLC, Platelet count, Reticulocyte count), bacteriological culture, chest X-ray, Blood chemistry and urine test were performed. Investigation reports were retrieved from the microbiology laboratory of CMCTH.
Blood culture was incubated at 370C over night and sub-culture on Mac Conkey agar was done. Growth negative culture was incubated for another 3 days watching for growth every $24 \mathrm{hrs}$. Records of positive blood culture cases were entered in separate record file. Cases were classified as EONS if they presented at the hospital at less than $72 \mathrm{hrs}$ of life and LONS if at or after $72 \mathrm{hrs}$ of life.

Besides clinical sepsis, the newborns were also admitted with the diagnosis of Neonatal jaundice, Respiratory distress syndrome, Birth asphyxia, Prematurity, Neonatal seizure, Congenital hydrocephalus, Omphalocele.

\section{RESULTS}

Table 1: Causes of neonatal admission $n=267$

\begin{tabular}{|l|l|l|l|}
\hline & Diagnosis & Total number & Percentage \\
\hline 1 & Clinical sepsis & 134 & 50.18 \\
\hline 2 & Neonatal Jaundice & 47 & 17.6 \\
\hline 3 & $\begin{array}{l}\text { Respiratory distress } \\
\text { syndrome }\end{array}$ & 22 & 8.2 \\
\hline 4 & Birth asphyxia & 19 & 7.1 \\
\hline 6 & Prematurity & 18 & 6.7 \\
\hline 7 & Neonatal seizure & 6 & 2.2 \\
\hline 8 & Congenital hydrocephalus & 4 & 1.49 \\
\hline 9 & Omphalocele & 1 & 0.37 \\
\hline
\end{tabular}

Clinical sepsis was present in 134 newborns accounting $(50.18 \%)$ of the total neonatal admission.

Blood cultures were done in all $(n=134)$ clinically suspected sepsis as a part of septic screening. Blood culture found to positive only in 10 cases $(7.46 \%)$.

E. Coli constituted the majority of the isolated organisms followed by Staph aureus. There were only a few cases of Klebsiella species. Pseudomonas and Clostridia group were not isolated in this study population. Most of the organisms were sensitive to Cefotaxime and Amikacin. However some patents were treated with Vancomycin and imipenum since clinical improvement was not seen with combination treatment with Cefotaxime and Amikacin. 


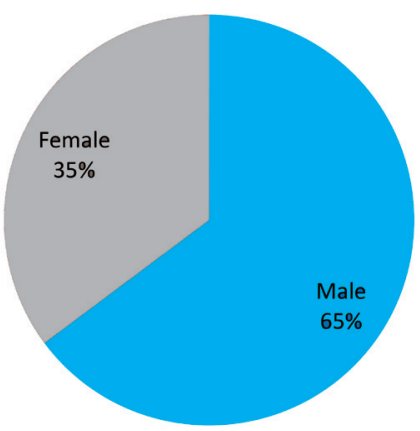

Figure 1: Sex wise distribution of NB $(n=267)$

There were 173(64.79\%) male and 94(34.21\%) female newborns admitted in this study period with the male to female ratio being almost 2:1. The reason of high number of male NB admission in the hospital could be because they give more preferences to the male sex than female in our society.

Table 2: Patients Characteristics $(n=267)$

\begin{tabular}{|l|l|l|}
\hline Gestation & Number & Percentage \\
\hline Term & 174 & 65.16 \\
\hline Preterm & 93 & 34.83 \\
\hline Mode of delivery & Number & Percentage \\
\hline NVD & 142 & 53.18 \\
\hline LSCS- ER & 105 & 39.32 \\
\hline LSCS-EL & 20 & 7.49 \\
\hline Birth weight (gm) & Number & Percentage \\
\hline$<1500$ & 17 & 6.36 \\
\hline $1500-2500$ & 128 & 47.95 \\
\hline$>2500$ & 122 & 45.69 \\
\hline
\end{tabular}

Among the NB admitted in the NICU during study period, $65 \%$ percent of newborn was born at term and 35 percent of them were preterm baby. Among the preterm, male 54(58\%) and female were $39(42 \%)$.

In sex distribution, in 1-3 years age group male $(n=211)$ are $140(66.3 \%)$ and female $71(33.64 \%)$ where as in 4-28 days age group $(\mathrm{n}=56)$ males were 33 and female 23 accounting $58.9 \%$ and $41 \%$ respectively.

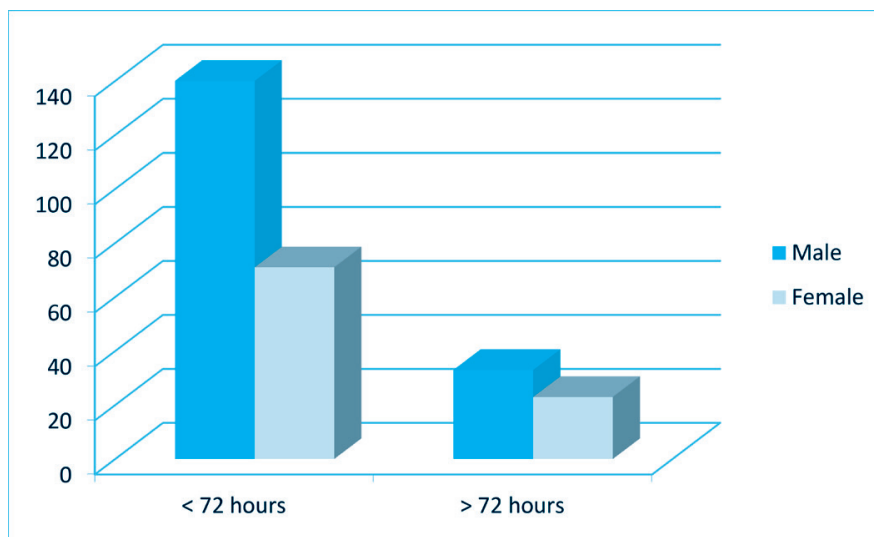

Figure 2: Age group by $\operatorname{Sex}(n=267)$

Table 3: Patients Age group $(n=267)$

\begin{tabular}{|l|l|l|}
\hline Age by hours of birth & Number & Percentage \\
\hline $1-3$ days (<72 hours) & 211 & 79.03 \\
\hline 4-28 days ( $>72$ hours) & 56 & 20.97 \\
\hline Total & 267 & 100 \\
\hline
\end{tabular}

Table 4: Clinical sepsis VS culture positive cases $=(134)$

\begin{tabular}{|l|l|l|}
\hline Diagnosis & Number & Percentage \\
\hline Clinical Sepsis & 134 & 50.18 \\
\hline Culture positive & 10 & 7.47 \\
\hline Culture negative & 124 & 92.53 \\
\hline
\end{tabular}

Blood glucose was evaluated in admitted newborns (202), but not in all as a routine test in the NICU unit. Generally, hypoglycemia tends to be more common in preterm and sick newborns, there is not much such trend seen in this study. Hypoglycemia was present only in $43 \mathrm{NB}(21.2 \%)$ preterm cases where as it was 40 $(19.8 \%)$ in term babies.

Table 4: Blood glucose level VS gestational age $(n=202)$

\begin{tabular}{|l|l|l|l|}
\hline Gestation & $<\mathbf{4 0} \mathbf{~} \mathbf{g} / \mathbf{d l}$ & $>\mathbf{4 0} \mathbf{~} \mathbf{g} / \mathbf{d l}$ & Total \\
\hline Term & 40 & 76 & 116 \\
\hline Preterm & 43 & 43 & 86 \\
\hline Total & 83 & 119 & 202 \\
\hline
\end{tabular}




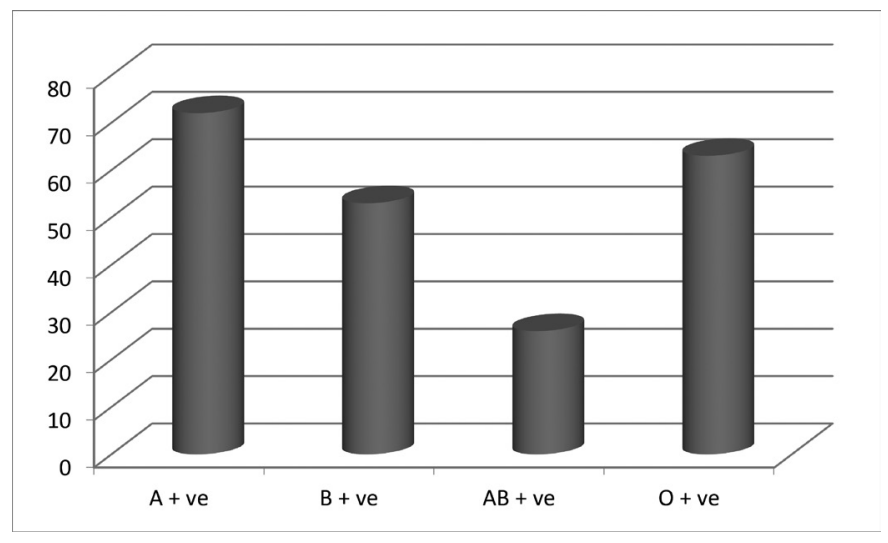

Figure 3: Blood group of newborn $(n=267)$

Blood grouping and cross matching was done in all NB admitted during the study period. Majority of newborns were found to be $\mathrm{A}+$ ive $72(27 \%)$ followed by $\mathrm{O}+$ ive in $63(23.6 \%)$ newborn. B+ive blood group was observed in $53 \mathrm{NB}$ accounting (19.85\%). Blood group $\mathrm{AB}+$ ive was found to be in 26 babies with $9.7 \%$. O-ive and A-ive blood group present in 1 in each newborn.

\section{CONCLUSION}

Neonatal sepsis is the most common causes of NICU admission in our centre even though there are other common cause of morbidity and mortality like asphyxia, RDS, neonatal jaundice, preterm deliveries and neonatal seizures. We must take strong initiatives to reduce the these causes of neonatal morbidity and mortality to meet millennium goal by 2015 .

\section{REFERENCES}

1. Bhutta ZA, Yusuff K. Neonatal sepsis in Karachi: Factors determining outcome and mortality. J Trop Pediatr 1997; 43: $65-70$.

2. Population division, Ministry of Health and Population; New ERA; ORC Macro International INC, Nepal Demographiv Health Survey 2011.

3. Bhutta ZA. Priorities in newborn care and development of clinical neonatology in Pakistan: where to know? J Coll physicians Surg Pak 1997;7:231-4.

4. Gurubacharya SM, Gurubacharya RL. An overview of neonatal admission at college of nmedical sciences. J Nepal Pediatr. Society 2007;27 (2):73-4.

5. Fort AL, Kothari MT, Abderrhim N. association between maternal, birth and newborn charecteristics and neonatal mortality in five Asian countries: Demographic and health research. August 2008;55

6. Stoll BJ. Infections of the neonatal infant. In; Behrman RE, Kllegman RM. Editors. Nelson Text book of Pediatrics, 18 edition. W.B. Saunders Company; 794-98.

7. Vergano S, Sharland M, Kazembe P, Mwansambo C,Health PT. Neonatal sepsis: an international perspective. Arch Dis. Child Fetal Neonatal 2005;90:F220-24.

8. Van den Hoogen A, Gerard LJ, Verboon- Maciolek MA, Fleer A. Krediet TG. Long term trends in the epidemiology of neonatal sepsis and antibiotic susceptibility of causative agents. Neonatology 2009; 97: 22-28. 